ARTICLE

https://doi.org/10.1057/s41599-019-0268-z

\title{
'I see you sharing, thus I share with you': indirect reciprocity in toddlers but not infants
}

\author{
Elena Nava ${ }^{1,2,3}$, Emanuela Croci $^{1} \&$ Chiara Turati ${ }^{1,2,3}$
}

\begin{abstract}
Human societies are organised around cooperative interactions, the origins and development of which have become a timely topic. In this study, we investigated the development of indirect reciprocity in 18-24-month-old toddlers, and infants aged 6 months, on a two-phase sharing task with non-familiar individuals. In the first phase, we observed whether infants and toddlers differentiated and manifested a preference toward an individual altruistically sharing or acting selfishly. In the second phase, infants and toddlers interacted with the same prosocial and antisocial individuals seen in the first phase, and we observed whether they were willing to share with one of the two. Indirect reciprocity was assessed as the match between the preferences for the prosocial individual in phase one, and the firstperson sharing in the second phase. Evidence showed that toddlers, but not infants, indirectly reciprocated the prosocial individual, suggesting that understanding of such a complex behaviour as indirect reciprocity may require prolonged experience in order to emerge.
\end{abstract}

\footnotetext{
${ }^{1}$ Department of Psychology, University of Milan-Bicocca, Milan, Italy. ${ }^{2}$ NeuroMi, Milan Centre of Neuroscience, Milan, Italy. ${ }^{3}$ MIBTEC: Mind and Behavior Technological Center, Milan, Italy. Correspondence and requests for materials should be addressed to E.N. (email: elena.nava@unimib.it)
} 


\section{Introduction}

he evolution of cooperation and altruism among non genetically related individuals represents one of the most astonishing and complex human behaviours. Among the different mechanisms that might promote cooperation (e.g., kin selection, direct reciprocity, see Nowak (2006) for a review), indirect reciprocity is the most intriguing because it accounts for behaviours, in which the donor does not receive (immediately) anything in return, following the rule: if individual $\mathrm{A}$ helps $\mathrm{B}$ while individual $\mathrm{C}$ observes the interaction between $\mathrm{A}$ and $\mathrm{B}, \mathrm{C}$ will be more willing to help A in the future (Boyd and Richerson, 1989; Nowak and Sigmund, 1998). Models of large-scale human cooperation (Nowak and Sigmund, 1998; Panchanathan and Boyd, 2004) have indeed shown that, in the long term, altruistic behaviour increases the reputation of the donor and thus the chance for him to obtain benefits in future encounters as a recipient.

Recent studies have revealed that precursors to cooperation mechanisms may be observed in infants as young as 3 months of age (Hamlin et al., 2010), particularly revealing that infants prefer individuals who behave altruistically than individuals who behave selfishly using different tasks (e.g., helping: Hamlin et al., 2010; Hamlin et al., 2007; Hamlin, Wynn et al., 2011; equal distribution of resources: Geraci and Surian, 2011; Meristo and Surian, 2014; Schmidt and Sommerville, 2011) and stimuli. That is, irrespective of whether infants see puppets (Hamlin et al., 2007; 2011), cartoons (Geraci and Surian, 2011; Meristo and Surian, 2013; 2014) or humans (Dunfield et al., 2011; Schmidt and Sommerville, 2011) performing the prosocial or antisocial behaviour, they tend to prefer prosocial over antisocial behaviours. While there is still debate on the reason why infants should prefer one behaviour over the other (see Paulus (2014) for a review), these studies converge on a substantial social characteristics of infants: they appear sensitive to the reputation of individuals and by the second year of age, they also use this information to decide how to respond to these individuals. For example, Dahl et al. (2013) showed that 26-month-old toddlers (but not 17-month-olds) looked longer to the antisocial individual during the familiarisation phase in which the child watched an individual rolling back the ball to another individual (prosocial interaction) or keeping the ball for himself (antisocial interaction). The longer looking times to the antisocial individual predicted helping behaviours directed towards the prosocial individual in a second phase, in which the toddler was asked to help either the prosocial or antisocial individual. Using puppets, Hamlin et al. (2011) showed that 19-23-month-old toddlers preferred to give a treat to a prosocial puppet, who previously helped another puppet opening a box rather than to an antisocial puppet who acted "mean", by jumping on top of the box. In the same study, the authors also tested 5-month-old and 6-month-old infants, and found that while younger infants solely evaluate the positive or negative effect on the recipient (i.e., they always prefer the prosocial behaviour irrespective of whether the recipient previously acted prosocially or antisocially), by 8 months of age infants take in consideration the reputation of the recipient to decide whether the latter should be treated "good" or "bad". These studies suggest that rudiments of indirect reciprocity can be observed in infancy, but the age at which it is observed may vary according to stimuli used.

It should be mentioned in a study that explicitly investigated indirect reciprocity in preverbal infants using a distributive task: Meristo and Surian (2013) showed 10-month-olds cartoons depicting two donors distributing two objects to two identical recipients. In one condition, the donor distributed equally the two objects ('fair donor), while in the other condition, the donor distributed the two objects to only one recipient ('unfair donor).
Following this familiarisation phase, the infants watched a human hand donating an object to either the fair ('expected reciprocity event') or unfair donor ('unexpected reciprocity event'). Infants watched longer the unexpected event, suggesting that they were expecting to observe the human hand donating to the fair donor, thus revealing that by 10 months of age, infants possess the complex social ability to expect that the fair actions of an individual should be indirectly reciprocated.

To the best of our knowledge, the study by Meristo and Surian (2013) is the sole that showed indirect reciprocity in the first year of age. Here, we sought to investigate whether this finding can be generalised to situations in which the social task is more complex - such as sharing-and requires a more prolonged developmental trajectory to emerge. Furthermore, differently from Meristo and Surian (2013) we were interested in investigating indirect reciprocity in a more first-person perspective; that is, we wanted to see whether this complex behaviour also emerges when it is the child to decide whether to reciprocate or not by directly interacting with the recipient. In the present study we therefore addressed the development of indirect reciprocity in a sharing task. Sharing is considered a prototypical prosocial behaviour, as it requires giving up own goods (Svetlova et al., 2010; Warneken and Tomasello, 2009) without the guarantee of reciprocation. Earlier studies have observed spontaneous offers of goods and toys in infants aged between 8 and 12 months of age, but this has occurred only when sharing was directed toward parents or familiar individuals (Hay, 1979; Hay and Murray, 1982). More recent and controlled studies have found that sharing becomes more and more spontaneous between 18 and 24 months of age (see e.g., Brownell et al., 2013). That is, while 24-month-old toddlers share without any communicative cue, 18-month-olds require more explicit support in order to exhibit such behaviour (e.g., a verbal request). This might be because sharing requires the ability to recognise inequality between oneself and another and to overcome the desire to keep the resource for oneself (Dunfield et al., 2011). It should be noted that "spontaneous sharing" should be considered so if it is prompted by only observing a need, without a verbal request to share.

Interestingly, to date no study has investigated whether sharing is at least understood at a cognitive level by younger infants. That is, while the act of sharing may have a protracted development because it is more costly than other behaviours, this does not preclude the fact that toddlers but also infants may be able to understand and prefer an individual who shares vs. non-shares. Observing that sharing is understood and preferred earlier than it is actually put in action would suggest that some prosocial behaviours dissociate between cognition and action, and, contrary to what Kohlberg suggested, cognitive understanding of prosocial behaviours does not always predict performance.

Furthermore, differently from Meristo and Surian (2013), we were interested in investigating indirect reciprocity in a more first-person perspective; that is, we wanted to see whether this complex behaviour also emerges when it is the child to decide whether to reciprocate or not by directly interacting with the recipient, i.e., decide whether to share or not with the individual who has previously behaved prosocially or antisocially. Hamlin et al. (2011) used a similar reasoning, by asking infants and toddlers to manually choose which character they preferred based on the reputation achieved by the same character on a previous familiarisation phase. However, the stimuli used by Hamlin et al. (2011) were puppets, and recent studies have failed to replicate such findings (Nighbor et al., 2017; Salvadori et al., 2015; Scarf et al., 2012). Thus, in the current study, we attempted to use human individuals to observe infants' and toddlers' proclivity to prosocial behaviour using different types of stimuli. We reasoned 
that, because especially infants are exposed and interact with real humans since birth, presenting humans could favour infants' and toddlers' understanding of the behaviour and motivate them to interact with them. Warneken and Tomasello (2006), for example, have shown that presented with humans, toddlers spontaneously act altruistically with unknown individuals. This pattern has shown to be robust both when the parent is present (and may encourage prosocial behaviour) or absent (Warneken and Tomasello, 2013). Even sharing appears to occur spontaneously in 15-month-olds when interacting with humans under naturalistic settings (Schmidt and Sommerville, 2011), which suggests that the use of such stimuli could promote prosocial understanding and behaviour even in younger infants.

In Experiment 1 we investigated indirect reciprocity in toddlers aged 18-24 by using a two-phase paradigm, in which they were required to both evaluate and act in first person in a sharing task. Toddlers were first asked to choose which of two human demonstrators (a prosocial and antisocial) they preferred (see Fig. 2 in the 'Method' section), following a brief video presentation of these human demonstrators behaving prosocially or antisocially (i.e., sharing or selfishly keeping the goods for her own, see Fig. 1 in the Method section). In a second phase, toddlers were given their own goods and interacted in first-person with the two human demonstrators seen in the videos, with whom they were requested to share their goods (see Fig. 3 in the Method section). We measured indirect reciprocity as a preference to share with the prosocial individual observed in the first phase.

Experiment 2 addressed whether precursors of indirect reciprocity can be observed in preverbal infants who, contrary to Meristo and Surian (2013), have to directly interact with a prosocial and antisocial character. To this end, we tested 6-montholds on a simplified version of the two-phase paradigm used for toddlers. We chose this age because previous studies had found prosocial behaviour in a helping task in 6-month-olds using a manual task (Hamlin et al., 2007), and we wanted to be able to compare our results with these studies, in particular assessing whether prosocial behaviour generalises with other tasks different from helping.

While the first phase was identical to the one used for toddlers, the second phase consisted in having the two human demonstrators offering a toy to the infant and observe to whom the infant would reach to. Thus, instead of directly assessing sharing, we observed infants' preference to take from one demonstrator or the other, based on their reputation. This was motivated by the fact that 6-month-olds still possess limited motor capacities that prevent them from performing any sharing. However, they can reach and grasp, so we looked at their preference to reach for a toy offered by one demonstrator over the other.
The use of human demonstrators wearing colourful suits was made to avoid that the preference for one demonstrator over the other could be made based on physical appearance (e.g., colour of hair) or involuntary facial expression of the demonstrator. Nevertheless, the suits did not cover the eyes, which has proven to influence prosocial judgement at younger ages (Hamlin et al., 2007).

Based on previous findings, we expected to see an overall preference for prosocial individuals and a preference to interact with the prosocial individual at both ages, thus providing the first evidence of early first-person indirect reciprocity in toddlers and the first evidence of sharing understanding in infants.

\section{Method}

\section{Experiment 1}

Participants. Thirty-eight 18-24-month-old toddlers (15 females, mean age $=20$ months, range: $17-24)$ were recruited from birth records provided by neighbouring cities near ${ }^{* * *}$ (blinded for the review process) via a written invitation that was sent to their parents. Five toddlers were discarded from the final sample because of inattention or fussiness. All children were Caucasian, and the socioeconomic status of the family was middle class. All children did not present neurological or cognitive deficits.

The parents of the children signed a written informed consent before starting the experiment. The study was approved by the Ethical Committee of the ${ }^{* * *}$ (blinded for the review process), and the study was conducted according to the principles expressed in the Declaration of Helsinki.

Materials, design and procedure. The study consisted in two phases, which we refer here to P1 (Phase 1) and P2 (Phase 2) for clarity. All toddlers were tested singularly at the ${ }^{* * *}$ (blinded for the review process), in a single quiet room which was divided in two adjacent spaces. The room was fully equipped for monitoring the response of the toddlers in both phases by videotaping their behaviour.

P1 was designed to assess the preference of toddlers between a prosocial and antisocial character in a sharing task. To familiarise the toddlers with the two behaviours, we presented them with two videos in which two human demonstrators, wearing colourful (red and green), fully covering suits, played the roles of the prosocial and antisocial character. The colour of the suit worn by the prosocial and antisocial human demonstrators was randomised across children. The prosocial and antisocial human demonstrators interacted with a recipient who always wore a black suit. The use of the suits had specific reasons: first, they covered (except for the eyes) all features that the child could use a.

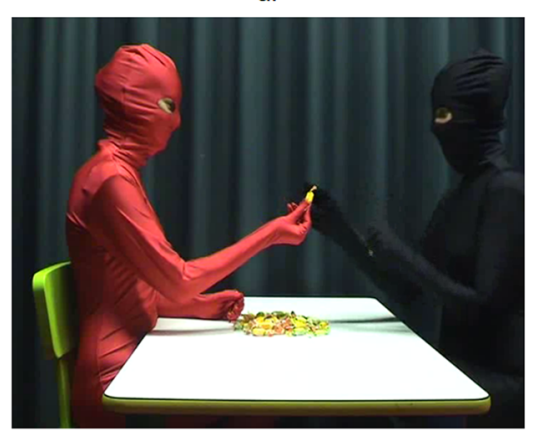

b.

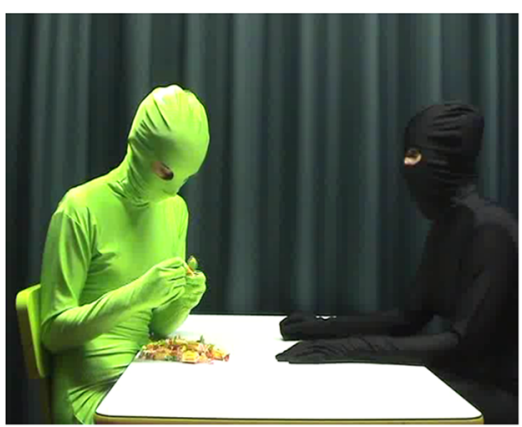

Fig. 1 Frames of the videos presented in P1. Panel a shows a scene of the prosocial character sharing with the recipient. Panel $\mathbf{b}$ shows a scene of the antisocial character selfishly keeping the goods for herself 
to make a choice. That is, a child could prefer a specific hair colour and length, or other features of the face. We also wanted to cover any facial expression that could be involuntary made by the experimenters and thus induce the preference of the child.

In one video, the prosocial human demonstrator sat at a table with candies placed on it and said: "These candies are yummy!". The recipient joined the prosocial demonstrator at the table and simply repeated the same sentence of the prosocial demonstrator, followed by the prosocial demonstrator sharing some candies with the recipient (see Fig. 1a). In the second video, the antisocial human demonstrator and the recipient acted and said the same words as in the first video ("These candies are yummy!"), with the only difference that after the words of the recipient, the antisocial human demonstrator took all candies close to herself, thus acting selfishly (see Fig. 1b). Note that the decision of having the human demonstrators speaking out a sentence was to attract the children's attention for a longer period, and to make them better understand the context.

There were overall 6 pairs of videos for a total of 12 videos: half of them depicted the prosocial behaviour, and the other half depicted the antisocial behaviour. To be included in the final sample, children had to watch at least two pairs of videos (overall 4 videos). The familiarisation stopped if the children watched 12 videos or if he/she looked away for $5 \mathrm{~s}$. Once the children were familiarised with the videos, they were brought to an adjacent, communicating space equipped with cameras to monitor their behaviour/response. The child was seated at a table and the experimenter presented her/him with two pillows (one next to each other) on which the picture of the prosocial and antisocial human demonstrator was printed. The experimenter would then ask: "Which one do you like?". The preference of the child towards one pillow or the other was considered as such if he/she showed an explicit action, for example, grasping one pillow, or putting the finger or the hand on top of it. The position of the two pillows was counterbalanced across children, so that half of the children saw the prosocial human demonstrator on the left, while the other half saw the same human demonstrator on the right side. The preference shown for either the prosocial or antisocial human demonstrator ended P1 (see Fig. 2 for a representative 18 month-old toddler choosing one character over the other). If the child did not choose after the experimenter asked for three times, the experimenter left the room after saying "goodbye" to the child.

P2 was designed to specifically assess whether children indirectly reciprocate the prosocial human demonstrator. Assuming a preference for the prosocial human demonstrator established in P1, P2 investigated whether children prefer to share their own goods with the prosocial human demonstrator. Specifically, we designed P2 to assess whether the child was able to take into consideration the reputation of the human demonstrator (observed in the familiarisation videos) and use this information to choose with whom to share. To this aim, at the beginning of P2, we placed a bowl full of candies on the table in front of the child. Then, the two human demonstrators entered the room, wearing the exact same suits worn in the videos and went to sit at the table next to each other in front of the child and repeatedly greeted the child by waving with one hand.

To assess indirect reciprocity, we measured requested sharing of the child after the two human demonstrators synchronously showed the child the right hand, as a sign of request. The request was only gestural and not verbal to avoid having a response that could reflect compliance (see Fig. 3 for a representative 18month-old toddler sharing).

\section{Results}

Scoring and reliability. The choices of the toddlers in P1 and P2 were videotaped and their responses coded by a rater unaware of the condition. That is, the rater judged if the child chose the red or the green demonstrator (i.e., the colour of the character depicted on the pillow in P1 and the colour of the character in P2). In P1, a preference was coded as such if the child manifested an explicit preference toward one of the two pillows, such as reaching or grasping. A similar coding scheme was applied in P2: sharing with one demonstrator was coded as such if the child gave or reached to the preferred character with the candy in his/her hand. A second rater, also unaware of the condition presented, coded $50 \%$ of the children, chosen randomly. Interrater reliability was $98 \%$.

Phase 1: Preference for prosocial or antisocial behaviour. Toddlers watched on average a total number of 9 videos during the familiarisation phase $(M=9.42 ; \mathrm{SD}=2.23)$. Thirty-two over 33 toddlers chose a pillow after watching the videos. The preference of the children was assessed with a two-tailed binomial probability test (see Hamlin et al., 2007; 2010; 2011; Dunfield and Kuhlmeier, 2010). Results showed that toddlers robustly preferred the prosocial over the antisocial human demonstrator, with 27 out of 32 choosing the prosocial over the antisocial human demonstrator, $p<0.001$, two-tailed binomial test, $95 \% \mathrm{CI}$ : $0.67-0.94$ (see Fig. 4 and Table 1 in the supplementary material for the responses provided by each toddler).

Phase 2: Sharing and indirect reciprocity score. In Phase 2, we observed the number of toddlers who shared with one of the two human demonstrators. Twenty-two out of 33 toddlers exhibited a response (note that the number of responders was nonetheless higher than the non-responders (binomial test, $p=0.04$ ). Among the 22 children that exhibited a response, 18 shared their goods with the prosocial than antisocial human demonstrator (see Fig. 4, $p=0.004$, two-tailed, 95\% CI: 0.61-0.93).

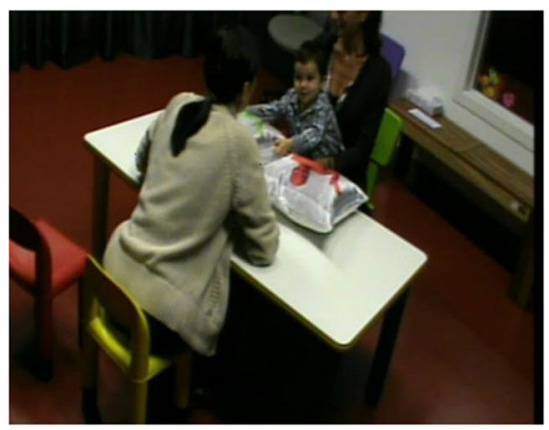

Fig. 2 Assessment of the preference between the prosocial and the antisocial character by a representative 18-month-old toddler. The toddler was asked "Which of the two do you prefer?" (left panel), and the choice was assessed by a clear reaching toward one of the two pillows (right panel) 

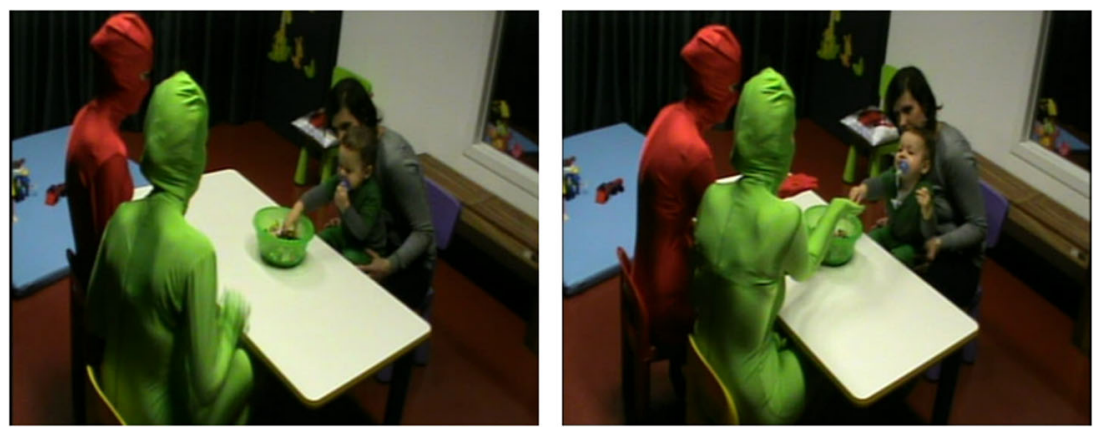

Fig. 3 Spontaneous sharing in an 18 month-old toddler. The two characters sat in front of the child (left panel) and did not produce any gesture unless the child did not show any behaviour. In that case (right panel), the two characters clearly manifested a sharing request by reaching out their hands to the child
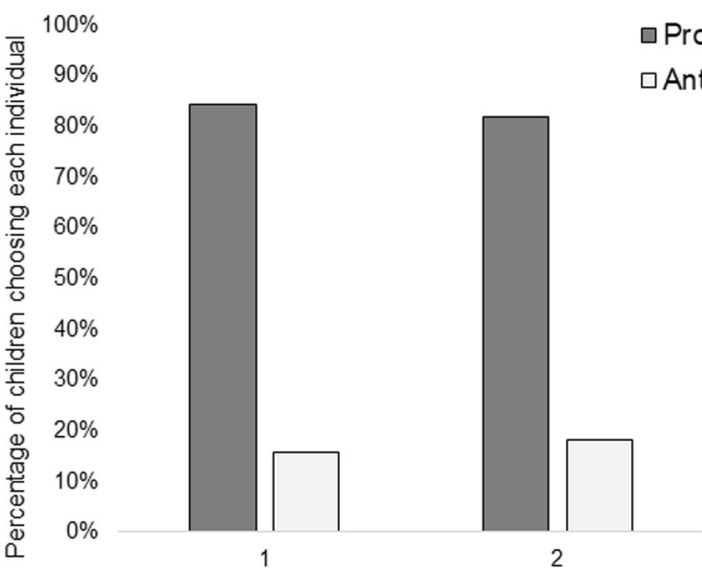

Fig. 4 Percentage and number of toddlers choosing either the prosocial or antisocial individual in P1 and P2. The graphs show that, both in the evaluation (left graph) and sharing (right graph) phase, toddlers preferred the prosocial over the antisocial character

To assess indirect reciprocity, we calculated a score based on the correspondence between P1 and P2; that is, we assigned "1" (i.e., congruency) to those toddlers who preferred the prosocial human demonstrator in $\mathrm{P} 1$ and shared with the same human demonstrator in $\mathrm{P} 2$, and " 2 " to those toddlers who did not chose congruently in the two phases (e.g., the ones who chose the prosocial human demonstrator in P1 but the antisocial human demonstrator in P2; see Table 1 of the supplementary materials for the scoring of each child). Furthermore, we also assigned " 0 " to those toddlers who did not exhibit a response in either Phase 1 or Phase 2.

In a first analysis, we included all toddlers: those who congruently chose the prosocial human demonstrator in both P1 and P2 $(N=16)$, toddlers who did not choose congruently between Phase 1 and $2(N=5)$, and those who did not prefer or share with the two human demonstrators in either Phase 1 or 2 $(N=12)$. However, this analysis did not reveal any difference across the three outcomes $\left(\chi^{2}=3.68, p=0.59\right)$.

Because this null result may have been influenced by the high number of toddlers who did not exhibit any response, we conducted a second analysis comparing toddlers who actually provided a response in both phases $(N=21)$. This analysis showed that 16 out of 21 toddlers congruently chose the prosocial human demonstrator ( $p=0.03$, two-tailed, $95 \%$ CI: $0.55-0.89$, see Fig. 5), thus revealing that indirect reciprocity may emerge in a selected sample.

Furthermore, to explore whether indirect reciprocity may be explained by factors such as age, gender and number of videos observed in P1, we conducted a logistic regression with the 21 toddlers included in the previous analysis. This analysis did not prove statistically significant, $\chi^{2}(8)=7.06, p=0.53$, and none of the factors explained indirect reciprocity (all $p>0.10$ ). Thus, age, gender and the exposure to more videos do not predict higher indirect reciprocity in toddlers.

Overall, the results of the indirect reciprocity score reveal that by the age of 18 months, toddlers' socio-moral evaluations of unfamiliar prosocial individuals are likely used to choose to share with these same individuals in a near future.

To observe whether there may be precursors to this type of complex cooperative behaviour, we tested a group of 6 month-old infants using the same task, however modifying the procedure particularly in P2 in order to accommodate the motor capabilities of younger infants. That is, instead of requesting a sharing, infants' proclivity to share with one human demonstrator over the other was assessed as preference to reach for the toy offered by the two human demonstrators.

\section{Experiment 2}

Participants. Twenty-seven, full-term 6-month-old infants (13 females, mean age $=6.8$ months, range: $6.5-7.0)$ were recruited from birth records provided by neighbouring cities near $* * *$ (blinded for the review process), via a written invitation that was sent to their parents. Six infants were discarded by the final sample due to inattention or fussiness. All children were Caucasian, and the socioeconomic status of the family was middle class. Testing took place in a quiet lab provided by the ${ }^{\star * *}$ (blinded for the review process).

The parents of the children signed a written informed consent before starting the experiment. The study was approved by the Ethical Committee of the ${ }^{\star \star \star}$ (blinded for the review process).

Materials, design and procedure. All infants underwent two-phases as toddlers did, with the following changes that were made to better suit the age: first, during the familiarisation phase, the human demonstrators used toys instead of candies because they are a more salient stimulus in comparison to candies (i.e., infants of that age have very little experience with solid food, and no experience with candies). Second, in P2 we did not measure requested sharing because of infants' limited motor abilities, but a preference to reach for two identical toys offered by the prosocial and antisocial demonstrator. That is, we observed a preference to interact with the prosocial vs. antisocial human demonstrator, evidenced by infants' reaching towards one demonstrator or the other.

All infants were tested singularly at the ${ }^{* *}$ (blinded for the review process), in a single quiet room, which was divided in two adjacent spaces. The room was fully equipped for monitoring the response of the infants in both phases by videotaping their behaviour. 


\section{Table 1 Distribution of responses of toddlers in experiment 1}

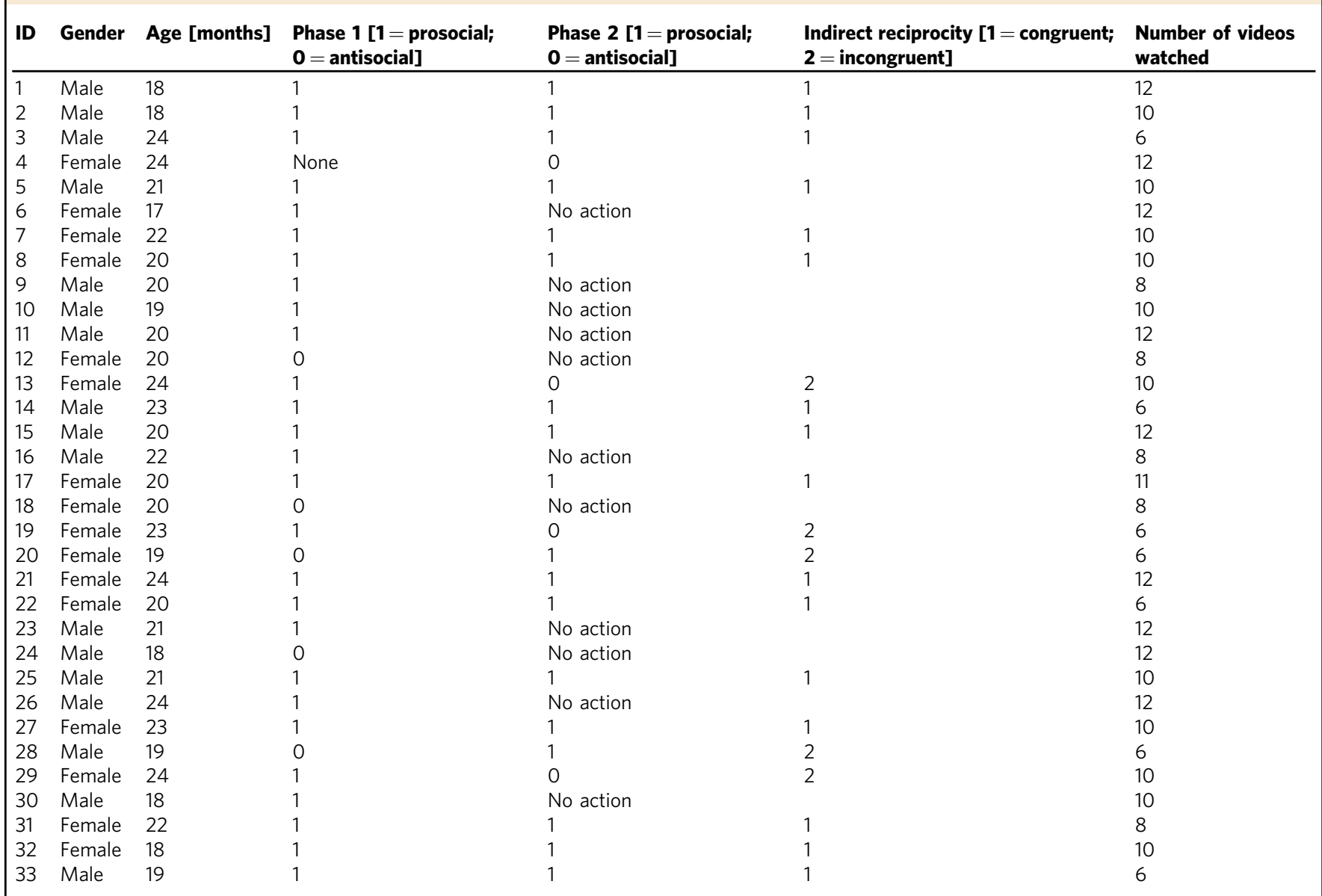

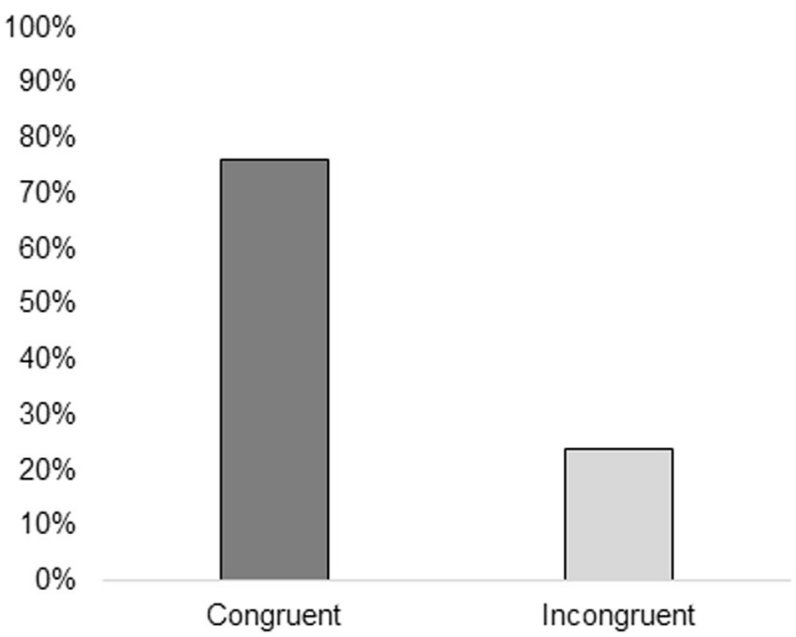

Fig. 5 Percentage of toddlers exhibiting indirect reciprocity. Indirect reciprocity was calculated as the congruency between P1 and P2, i.e., choosing and also sharing with a prosocial character. On the contrary, incongruent responses were those in which children preferred the prosocial character only in one of the phases

\section{Results}

Phase 1: Preference for prosocial or antisocial behaviour. Results of P1 showed that infants watched on average 10.38
$(\mathrm{SD}=1.86)$ videos. When asked to choose a pillow, 20 out of 21 infants showed a response. One did not choose any of the pillows. However, analyses conducted on 20 infants did not show any preference for the prosocial over the antisocial human demonstrator (11 out of 20 chose the prosocial actor, $p=0.83$, twotailed, 95\% CI: $0.34-0.74$, see Table 2 for a detail of the responses provided by each infant).

Phase 2: Reaching behaviour. In P2, only 18 out of 21 infants exhibited a response. Of these 18, 7 accepted the offer of the prosocial human demonstrator, thus, as in $\mathrm{P} 1$, no preference for the prosocial human demonstrator was observed $(p=0.48$, twotailed, 95\% CI: 0.20-0.61). Because results of infants in both P1 and P2 showed that no selective preference for the prosocial human demonstrator was observed, an analysis on indirect reciprocity would not be reasonable. However, as reported in Table 2, it is worth noting that 3 infants congruently chose the prosocial human demonstrator, and other 3 infants congruently chose the antisocial human demonstrator. Interestingly, 11 infants chose incongruently, i.e., they chose either the prosocial or antisocial human demonstrator in $\mathrm{P} 1$ and the opposite character in P2.

Overall, the results of the 6-month-old infants suggest that, at least in a sharing task, and using human demonstrators, infants do not prefer a prosocial over an antisocial individual as assessed in both phases of the task.

Finally, we compared infants' data with the toddlers' outcome. Chi-Square tests showed that in both $\mathrm{P} 1\left(\chi^{2}=5.40, p=0.02\right)$ and P2 $\left(\chi^{2}=7.78, p=0.005\right)$, toddlers showed a higher preference for 
Table 2 Distribution of responses of infants in experiment 2

\begin{tabular}{|c|c|c|c|c|c|c|}
\hline ID & Gender & Age [days] & $\begin{array}{l}\text { Phase } 1[1=\text { prosocial; } \\
0=\text { antisocial }]\end{array}$ & $\begin{array}{l}\text { Phase } 2[1=\text { prosocial; } \\
0=\text { antisocial }]\end{array}$ & $\begin{array}{l}\text { Indirect reciprocity }[1=\text { congruent; } \\
2=\text { incongruent; } 0=\text { no response }]\end{array}$ & $\begin{array}{l}\text { Number of videos } \\
\text { watched }\end{array}$ \\
\hline 1 & 1 & 222 & 1 & 0 & 2 & 10 \\
\hline 2 & 2 & 204 & 1 & 0 & 2 & 8 \\
\hline 4 & 1 & 195 & 0 & 1 & 2 & 8 \\
\hline 5 & 2 & 201 & 0 & No response & 0 & 12 \\
\hline 6 & 1 & 188 & 0 & 0 & 1 (antisocial) & 8 \\
\hline 9 & 1 & 184 & 0 & 0 & 1 (antisocial) & 12 \\
\hline 10 & 2 & 209 & 0 & No response & 0 & 8 \\
\hline 11 & 2 & 196 & 1 & 0 & 2 & 12 \\
\hline 12 & 1 & 204 & 1 & No response & 0 & 12 \\
\hline 13 & 1 & 212 & 0 & 1 & 2 & 8 \\
\hline 14 & 1 & 200 & 1 & 0 & 2 & 12 \\
\hline 20 & 1 & 216 & 0 & 0 & 1 (antisocial) & 10 \\
\hline 21 & 2 & 153 & 1 & 1 & 1 & 12 \\
\hline
\end{tabular}

the prosocial over the antisocial human demonstrator in comparison with infants.

\section{Discussion}

The ability to evaluate the behaviours of others, and particularly the ability to judge the good or bad reputation of other individuals is crucial to adjust one's own behaviour to benefit from future interactions. For children, the development of such skill could be particularly helpful in driving their preference to interact (or not) with new encounters.

In this study, we showed that by 18 months of age, toddlers choose to indirectly reciprocate prosocial over antisocial others, based on sole observations of their previous actions. We have thus shown that early in development, (positive) reputation is already taken as a reliable cue to determine future interactions. This study adds new evidence to the notion that toddlers start very early to form complex social thinking and that different information are taken into consideration to intend the goals of others and thus likely obtain the greatest future benefits.

However, it should be noted that the first analysis conducted on the whole sample (i.e., including the toddlers who also did not exhibit any response) did not prove significant; that is, our findings can only be applied to a smaller set of the sample who was able to conclude the task. Indeed, $33 \%$ of toddlers did not exhibit any response in Phase 2. Although a similar proportion of non-responders has also been found in other studies (Dunfield et al., 2011; Schmidt and Sommerville, 2011), and although it is very difficult to determine the nature of a non-behaviour (as it could stem from a number of different reasons, e.g., not having understood the task, the character's request, distraction, inattention, or even struggle to decide what to do), we cannot exclude that some toddlers simply do not engage in (indirect) reciprocal sharing. While it is difficult to disentangle whether it is indirect reciprocity or sharing itself to inhibit the response, there is evidence showing that toddlers only engage in spontaneous sharing if the recipient provides explicit communicative cues about his/ her needs (Brownell et al., 2009). In our study, we did not want to use communicative cues, but only used gestural cues (i.e., pointing), because we wanted to avoid toddlers to comply with the recipient's wishes. That is, the prosocial actions observed in studies in which the request was made verbally explicit (Brownell et al., 2009; Schmidt and Sommerville, 2011) might have simply reflected the tendency of young children to obey to adults, but not a genuine prosocial behaviour. Interestingly, studies have reported a difficulty in adhering to prosocial principles, even at later ages when prosocial norms are well understood and specifically in sharing tasks. For example, Smith et al. (2013) have shown that 3year-old children know that they should share equally, but fail to do so at least until 7-8 years of age. This study seems to support our finding, which suggest that the understanding of sharing actions is understood and preferred by 18 months of age (P1), but the action of sharing (as seen in P2) emerges later in development, likely when children start adhering more coherently to their moral norms/principles, thus producing actions that are more in line with their understanding and 'approval' of the norm.

Studies have also revealed that it is not before 5-6 years of age that children spontaneously reciprocate recipients who have previously shown prosocial behaviour toward a third party (KatoShimizu et al., 2013), thus supporting the possibility that indirect reciprocity may be a late developing aspect of prosocial behaviour. Interestingly, the concept of reputation, which is strongly linked to indirect reciprocity, seems to emerge around 5-6 years of age too. A study has indeed shown that 5-year-old children tended to share more resources with another child when a third party watching them could reciprocate later (Engelmann et al., 2013). This behaviour was even more explicit when the interactions occurred with ingroup than outgroup members, overall suggesting that children not only acknowledge the importance of reputation by age 5 , but are aware that it may be more functional among familiar than unfamiliar individuals.

It should be noted that the second analysis we conducted on those toddlers who decided to engage with either a prosocial or antisocial human demonstrator revealed that at least a subset of toddlers indirectly reciprocated the sharing, corroborating previous findings showing that toddlers are strong reciprocators (Dunfield and Kuhlmeier, 2010). Indeed, Dunfield and Kuhlmeier (2010) showed that by 21 months of age, toddlers selectively help those individuals who have previously shown the willingness to help others, thus suggesting that toddlers take in consideration the reputation of their recipients. Our findings integrate the study 
of Dunfield and Kuhlmeier (2010) and others (e.g., Dahl et al., 2013; Hamlin et al., 2011; Schmidt and Sommerville, 2011), by showing that infants selectively choose their future partner only by observing their prosocial behaviour. Toddlers can detect who is "good" irrespective of whether the prosocial individual has shared with them previously, and use this information to decide with whom to share when it comes to first-person interactions.

Our data do not allow to conclusively state the specific reason that motivated toddlers to share with the prosocial over the antisocial individual. That is, the action observed was of indirect reciprocity, but whether it was done for sense of fairness or coherence between the response provided in P1 and P2, is difficult to disentangle. In other words, studies have shown that sense of fairness emerges very early in development (Geraci and Surian, 2011) and during distributive actions, infants commonly prefer individuals who distribute equally over individuals who distribute unequally. Thus, it could be claimed that both infants and toddlers in our study saw that the prosocial demonstrator was giving away more candies during the familiarisation phase and wanted to re-establish equality in P2. In infants, this might have translated into taking the offer from the antisocial actor (because he presumably had more toys), and into sharing with the prosocial actor for the toddlers.

An alternative explanation for the responses of the infants and toddlers could be that, because they were asked to choose which actors they preferred in P1, this drove their sharing behaviour in $\mathrm{P} 2$. That is, their behaviour was an act of coherence, but not motivated by real altruistic motivation, and future studies should disentangle this issue. However, while this may apply to toddlers, it should be noted that most infants chose different actors in P1 and $\mathrm{P} 2$, thus suggesting a preference for the novelty in P2. While the current study cannot exclude either of the alternative explanations, it should be noted that to date, the motivations behind prosocial behaviour in young children are still very speculative (see Paulus (2014) for a recent review).

Finally, it should be discussed why 6-month-old infants did not manifest any preference for the prosocial individual, which may sound in contradiction with previous findings (Hamlin et al., 2007; 2011). First, it should be noted that the studies Hamlin et al. $(2007 ; 2010$; 2011) presented the infants a helping behaviour, while we presented infants with a sharing task. It could well be that the two tasks require different cognitive abilities, which do not develop in parallel. This is indeed supported by studies that have shown that while helping behaviours emerge early in infancy and even in non-human primates (Dunfield et al., 2011; Warneken and Tomasello, 2006), sharing behaviours are observed only starting from the second year of life (Brownell et al., 2013; though see Schmidt and Sommerville, 2011). Our findings support this point, in that the toddlers tested in our study showed difficulties in the sharing task. This difference may stem from the different nature and cost of the two behaviours. In other words, while helping mostly consumes time, sharing requires, first, the ability to recognise that there is an inequality; then, the will to donate one's own goods. In this regard, it could be that sharing, even if performed by third parties, may be cognitively too difficult for 6-month-olds. Why should infants be facilitated in understanding helping, but not sharing actions? Paulus (2014) has suggested that there are four models explaining the emergence of prosocial behaviour early in life. Among these, one model suggests that some prosocial behaviours may reflect the understanding of goal-directed actions, such as helping. In the typical helping tasks (Dunfield et al., 2011; Hamlin et al., 2007), puppets or geometrical shapes clearly show their struggle towards a goal (e.g., trying to open a box or climb a hill), and the prosocial character helps achieving this goal. On the contrary, in our sharing task the action was very dry, with the recipient showing very little wish to receive something. Thus, it could be speculated that early in life infants do not prefer prosocial individuals themselves, but their capability of fulfilling a goal-directed action. In support to this claim, studies have shown that during the first year of life, infants not only understand goal-directed actions (Biro et al., 2007), but also that goals exist irrespective of the actions that result from them (Csibra et al., 1999). In this vein, it could be claimed that helping more clearly depicts a goal-directed action, and that younger infants prefer prosocial individuals because they are more successful in their attempts.

Taken together, our findings suggest that indirect reciprocity does not emerge before 18 months of age, as assessed by firstperson interactions. Our findings add new knowledge to the view that complex social behaviours develop during the second year of life (Meristo and Surian, 2014; Paulus, 2014), and could be particularly helpful in driving the preference of toddlers to interact (or not) with new encounters. Our findings in 6month-old infants, instead of challenging previous studies that have documented early development of prosocial behaviour (Hamlin et al., 2007; 2011), integrate them by suggesting that specific tasks, such as sharing, may require more first-person experience in order to be evaluated.

\section{Data availability}

All data generated or analysed during this study are included in this published paper.

Received: 4 January 2019 Accepted: 7 May 2019

Published online: 04 June 2019

\section{References}

Biro S, Csibra G, Gergely G (2007) The role of behavioral cues in understanding goal-directed actions in infancy. Prog Brain Res 164:303-322

Boyd R, Richerson PJ (1989) The evolution of indirect reciprocity. Soc Netw 111 (6685):213-236

Brownell CA, Iesue SS, Nichols SR, Svetlova M (2013) Mine or yours? Development of sharing in toddlers in relation to ownership understanding. Child Dev 84(3):906-920

Brownell CA, Svetlova M, Nichols S (2009) To share or not to share: when do toddlers respond to another's needs? Infancy 14(1):117-130

Csibra G, Gergely G, Bíró S, Koos O, Brockbank M (1999) Goal attribution without agency cues: the perception of 'pure reason' in infancy. Cognition 72 (3):237-2267

Dahl A, Schuck RK, Campos JJ (2013) Do young toddlers act on their social preferences? Dev Psychol 49(10):1964-1970

Dunfield KA, Kuhlmeier VA (2010) Intention-mediated selective helping in infancy. Psychol Sci 21(4):523-527

Dunfield K, Kuhlmeier VA, O'Connell L, Kelley E (2011) Examining the diversity of prosocial behavior: Helping, sharing, and comforting in infancy. Infancy 16(3):227-247

Engelmann JM, Over H, Herrmann E, Tomasello M (2013) Young children care more about their reputation with ingroup members and potential reciprocators. Dev Sci 16(6):952-958

Geraci A, Surian L (2011) The Development roots of fairness: infants' reactions to equal and unequal distributions of resources. Dev Sci 14(5):1012-1020

Hamlin JK, Wynn K, Bloom P (2007) Social evaluation by preverbal infants. Nature 450(7168):557-559

Hamlin JK, Wynn K, Bloom P (2010) Three-month-olds show a negativity bias in their social evaluations. Dev Sci 13:923-929

Hamlin JK, Wynn K, Bloom P, Mahajan N (2011) How infants and toddlers react to antisocial others. Proc Natl Acad Sci USA 108(6):19931-19936

Hay D (1979) Cooperative interactions and sharing between very young children and their parents. Dev Psychol 15(6):647-658

Hay DF, Murray P (1982) Giving and requesting: social facilitation of infants' offers to adults. Infant Behav Dev 5(2-4):301-310

Kato-Shimizu M, Onishi K, Kanazawa T, Hinobayashi T (2013) Preschool children's behavioral tendency toward social indirect reciprocity. PLoS ONE 8(8): e70915

Meristo M, Surian L (2013) Do infants detect indirect reciprocity? Cognition 129 (1):102-113 
Meristo M, Surian L (2014) Infants distinguish antisocial actions directed towards fair and unfair agents. PLoS ONE 9(10):e110553

Nighbor T, Kohn C, Normand M, Schlinger H (2017) Stability of infants' preference for prosocial others: Implications for research based on single-choice paradigms. PLoS ONE 12(6):e0178818

Nowak MA (2006) Five rules for the evolution of cooperation. Science 314 (5805):1560-1563

Nowak MA, Sigmund K (1998) Evolution of indirect reciprocity by image scoring. Nature 393(6685):573-577

Panchanathan K, Boyd R (2004) Indirect reciprocity can stabilize cooperation without the second-order free rider problem. Nature 432(7016):499-502

Paulus M (2014) The emergence of prosocial behavior: why do infants and toddlers help, comfort, and share? Child Dev Perspect 8(2):77-81

Salvadori E, Blazsekova T, Volein A, Karap Z, Tatone D, Mascaro O, Csibra G (2015) Probing the strength of infants' preference for helpers over hinderers: two replication attempts of Hamlin and Wynn (2011). PLoS ONE 10(11): e0140570

Scarf D, Imuta K, Colombo M, Hayne H (2012) Social evaluation or simple association? Simple associations may explain moral reasoning in infants. PLoS ONE 7(8):e42698

Schmidt MF, Sommerville JA (2011) Fairness expectations and altruistic sharing in 15-month-old human infants. PloS ONE 6(10):e23223

Smith CE, Blake PR, Harris PL (2013) I should but I won't: Why young children endorse norms of fair sharing but do not follow them. PloS ONE 8(3): e59510

Svetlova M, Nichols SR, Brownell CA (2010) Toddlers' prosocial behavior: from instrumental to empathic to altruistic helping. Child Dev 81(6):1814-1827

Warneken F, Tomasello M (2006) Altruistic helping in human infants and young chimpanzees. Science 311(5765):1301-1303

Warneken F, Tomasello M (2009) The roots of human altruism. Br J Psychol 100 (3):455-471

Warneken F, Tomasello M (2013) Parental presence and encouragement do not influence helping in young children. Infancy 18(3):345-368

\section{Acknowledgements}

We would like to thank Camilla Tognato and Giulia Cavandoli for helping to recruit and test the infants and toddlers involved in the study.

\section{Additional information}

Competing interests: The authors declare no competing interests.

Ethical approval: The Ethical Committee of the University of Milan-Bicocca (Italy) approved the studies presented in the manuscript. Informed consent was obtained by the legal guardians of all infants and toddlers before they took part in the studies.

Reprints and permission information is available online at http://www.nature.com/ reprints

Publisher's note: Springer Nature remains neutral with regard to jurisdictional claims in published maps and institutional affiliations.

\section{(c) (i)}

Open Access This article is licensed under a Creative Commons Attribution 4.0 International License, which permits use, sharing, adaptation, distribution and reproduction in any medium or format, as long as you give appropriate credit to the original author(s) and the source, provide a link to the Creative Commons license, and indicate if changes were made. The images or other third party material in this article are included in the article's Creative Commons license, unless indicated otherwise in a credit line to the material. If material is not included in the article's Creative Commons license and your intended use is not permitted by statutory regulation or exceeds the permitted use, you will need to obtain permission directly from the copyright holder. To view a copy of this license, visit http://creativecommons.org/ licenses/by/4.0/.

(C) The Author(s) 2019 